\title{
Les courbes et les droites. Patience en Allemagne et impatience en France à l'époque révolutionnaire. L'interprétation de Jean Jaurès
}

The curves and the lines. Patience in Germany and impatience in France during the Revolutionary period. The interpretation of Jean Jaurès

Éric Guillet

\section{OpenEdition \\ Journals}

\section{Édition électronique}

URL : https://journals.openedition.org/ahrf/11688

DOI : 10.4000/ahrf.11688

ISSN : 1952-403X

\section{Éditeur :}

Armand Colin, Société des études robespierristes

\section{Édition imprimée}

Date de publication : 1 juin 2010

Pagination : 173-196

ISBN : 978-2-200-92632-8

ISSN : 0003-4436

\section{Référence électronique}

Éric Guillet, « Les courbes et les droites. Patience en Allemagne et impatience en France à l'époque révolutionnaire. L'interprétation de Jean Jaurès », Annales historiques de la Révolution française [En ligne], 360 | avril-juin 2010, mis en ligne le 01 juin 2013, consulté le 23 avril 2022. URL : http:// journals.openedition.org/ahrf/11688; DOI : https://doi.org/10.4000/ahrf.11688 


\title{
LES COURBES ET LES DROITES. PATIENCE EN ALLEMAGNE ET IMPATIENCE EN FRANCE À L'ÉPOQUE RÉVOLUTIONNAIRE. L'INTERPRÉTATION DE JEAN JAURÈS
}

\begin{abstract}
Dans son Histoire socialiste de la Révolution française, Jaurès consacre à "La Révolution et l'Europe» cinq cents pages dont la moitié à "La Révolution et l'Allemagne». Cette approche comparatiste foncièrement ouverte oppose, certes de façon traditionnelle depuis $\mathrm{M}^{\mathrm{me}}$ de Staël, une culture allemande apolitique, cultivant l'attente patiente de jours meilleurs, à une culture française très politisée de l'action immédiate, mais croit, malgré une certaine préférence ethnocentrique pour l'impatience révolutionnaire française, qu'un rapprochement entre les deux cultures est possible et même souhaitable. Cette comparaison est aussi pour notre historien philosophe l'occasion de percer le secret de l'origine des révolutions. Mais on peut aussi reprocher à Jaurès que l'Allemagne qu'il dépeint, même s'il en éclaire le contexte politique et, chose nouvelle en 1900, économique, se réduit peut-être trop à celle, certes substantielle, des grands penseurs (Kant, Schiller, Forster, Fichte...) et néglige le pays "profond». Nous nous proposons ici d'analyser, dans ses grands traits, avec ses points forts et ses faiblesses, l'image que Jaurès donne de l'Allemagne de la fin du xvıII siècle.
\end{abstract}

Mots-clés: Allemagne et Révolution, mentalité allemande, mentalité française, comparatisme, transferts culturels. 
«Étudie l'historien avant de commencer à étudier lesfaits »'. L'historien anglais Carrrappelaitainsiquelesfaits sont toujours perçus réfractés par le regard que les hommes portent sur eux. Les interprétations qu'on peut en donner sont variées. La Révolution française n'échappe pas à cette loi et a donné lieu à maintes images divergentes chez les historiens. En 1900, I'histoire « socialiste » proposée par Jaurès renoue avec la lignée des histoires "démocrates » (Louis Blanc, Lamartine, Michelet) et constitue une césure dans la façon d'écrire la Révolution puisqu'elle a inspiré au $x x^{e}$ siècle l'œuvre de Mathiez, Lefebvre, Labrousse et Soboul.

Les différences entre toutes ces images de la Révolution tiennent à des proportions et des éclairages différents que nous voudrions faire apparaître dans l'image « socialiste » que Jaurès, dans son histoire de la Révolution, donne de la mentalité allemande entre 1789 et 1794.

La partie (1789-1794) de l'Histoire socialiste de la Révolution française écrite par Jaurès fut publiée à partir de février 1900. C'est avec L'Armée nouvelle sans doute le plus connu de ses livres. D'ailleurs, l'une des priorités de la Société d'études jaurésiennes, après sa création en 1959, fut d'encourager une réédition critique de l'œuvre à laquelle nous nous référons ${ }^{2}$.

Cet ouvrage, fruit des recherches menées par Jaurès sur la Révolution française depuis $1892^{3}$, n'eut pas le succès escomptét. L'œuvre ne plut ni au grand public « populaire » auquel elle s'adressait en priorité,

(1) Edward Hallett CarR, Was ist Geschichte? (Qu'est-ce que l'histoire?), Stuttgart, Kohlhammer, 1972, p. 23.

(2) Jean JAURĖs, Histoire Socialiste de la Révolution française, sept tomes dont le tome IV sur l'Allemagne. Édition revue et annotée par Albert Soboul, préfacée par Ernest Labrousse et présentée par Madeleine Rebérioux, Paris, Messidor, Éditions Sociales, 1983-1986. Désormais, nous ne citerons plus cet ouvrage que sous le sigle HSRF. En 2006, les Éditions Tallandier ont publié des morceaux de la HSRF choisis par Lorraine DE PLUNKETT et présentés par Max Gallo : Jean Jaurès. Louis XVI. Le procès de la royauté. Des 310 pages, six (p. 241-246) offrent des extraits du tome IV.

(3) Cela ressort d'une lettre de Jaurès au social-démocrate allemand Bernstein du 25 janvier 1902. Citée dans Madeleine RebérIoux, "Jaurès, historien de la Révolution française » dans Dommanget, Godechot, Labrousse, Rebérioux, Soboul etc...(éd.), Jaurès, historien de la Révolution française, Castres, Centre National et Musée Jaurès, 1989, p. 40. Pour plus de renseignements sur Jaurès historien de la Révolution, on se référera à Franco VENTURI, " Jaurès historien » dans Franco VentuRI (éd.), Historiens du xx siècle, Genève, Librairie Droz, 1966, p. 5-60 et, plus récemment, à Valérie Lecoulant, Jaurès, historien de la Révolution française, Montreuil, Musée de l'histoire vivante, 1993.

(4) Cf. Gilles Candar, "L'accueil de l'Histoire Socialiste de la Révolution », Cahiers Jaurès $n^{\circ} 1,1991$, p. 95. 
ni aux leaders socialistes, ni vraiment aux historien ${ }^{5}$, et les revues généralistes, même socialistes, observèrent à son égard un « silence presque absolu $»^{6}$. Finalement chère pour les classes populaires et présentée sousuneformepeupédagogique (pas de divisionsen parties introduites par Mathiez dans son édition de 1922, très longues citations), elle ne satisfait pas non plus aux critères universitaires (pas de références, style parfois trop oratoire ou lyrique) et Aulard, Mathiez et Andler, malgré leurs compliments, ne manquent pas de pointer du doigt les défauts de l'ouvrage. Dans une lettre de 1916 à Lévy-Bruhl, le grand germaniste socialiste Charles Andler déplore, dans le chapitre sur l'histoire des idées allemandes, d'importantes erreurs de détail 7 . Elles provenaient surtout de confusions de noms ${ }^{8}$ et ont pour la plupart été corrigées par Mathiez en 1922.

Ce sont précisément les pages de Jaurès sur l'Allemagne qui ont fourni la matière de ce que Gilles Candar appelle « la seule attaque qui ne soit pas insignifiante venue de la gauche ${ }^{9}$, celle venant de l'une des personnalités de la social-démocratie allemande, Franz Mehring. Historien, brillant théoricien marxiste, proche de l'extrême gauche du parti autour de Rosa Luxemburg et de Karl Liebknecht, il a publié en 1893 La légende de Lessing qui se veut « l'application sans réserve de la méthode matérialiste de recherche que Marx et Engels ont mise au point d'une manière tellement évidente $»^{10}$. Cette dévotion vis-à-vis de la « science » marxiste a indisposé Jaurès qui, dans son chapitre sur l'Allemagne, critique l'étroitesse du «matérialisme économique » que Mehring aurait appliqué mécaniquement. En effet, Jaurès pense que la réalité profonde déborde toujourslesfaits en général, économiques et sociauxen particulier $^{11}$. Mis en cause par le Français, le marxiste allemand répliquera dès février 1903 dans la revue Die Neue Zeit par l'article ironique Pour le roi de Prusse (en français dans le texte allemand). Il y accuse I'historien

(5) Cf. Jacques Godechot, Un jury pour la Révolution, Paris, Robert Laffont, 1974, p. 281.

(6) Gilles CANDAR, op. cit., p. 91.

(7) Cf. la présentation de cette lettre par Gilles Candar dans « Une lettre d'Andler sur les CFuvres de Jaurès. Une polémique qui ne passe pas... », Cahiers Jaurès, n 171, janvier-mars 2004, p. 49-55.

(8) Ibid., p. 53.

(9) Id., p. 91.

(10) Franz Mehring, Die Lessing-Legende, Frankfurt-Berlin-Wien, Ullstein, p. 5 (traduit par nous).

(11) Cf. Bruno Antoninl, "Jaurès historien de l'avenir: gestion philosophique d'une "méthode socialiste" ", Histoire socialiste de la Révolution française, AHRF, n 337, juillet/septembre 2004, p. 117-142. 
Jaurès de travailler trop vite (selon la « méthode teuf-teuf », dit-il), donc superficiellement ${ }^{12}$.

\section{Une histoire particulière de la Révolution}

L'histoire de la Révolution de Jaurès se distingue des précédentes histoires françaises de la Révolution. D'abord, elle repose sur des documents de première main peu ou pas exploités par ses prédécesseurs, par exemplelesœuvresdepenseurscommeBarnave,Burke,Fichteou Forster. Ensuite, l'œuvre accorde une importance fondamentale aux rapports de propriété et aux faits économiques, négligés par les prédécesseurs de Jaurès ${ }^{13}$ à cause principalement de l'indigence des documents dans ce domaine à laquelle le leader socialiste remédiera ${ }^{14}$. Enfin, à la différence des précédentes histoires françaises ${ }^{15}$ de la Révolution centrées sur la France, elle consacre tout le tome IV, La Révolution et l'Europe, à l'attitude face à la Révolution française de trois pays européens : I'Allemagne, la Suisse et l'Angleterre. Ce ne sont pas les relations diplomatiques qui intéressent ici Jaurès (Albert Sorel était en train de terminer de les traiter dansunouvragevolumineux ${ }^{16}$, mais, approcheculturelleinhabituelleen 1903, la vision du monde diffuse dans ces trois pays en 1789: "Que pense vraiment le monde de notre révolution $»^{17}$ ?

(12) Il se trouve en allemand dans les œuvres complètes de Mehring publiées en RDA : Franz Merring, "Pour le roi de Prusse », Gesammelte Schriften, Bd.9, Berlin (Ost), Dietz, 1963, p. 386-400. Il a été publié en français dès mai 1903 dans le $n^{\circ} 119$ de la revue Le Mouvement socialiste désireuse de rompre avec Jaurès (cf. Gilles CANDAR, op. cit., p. 91). Dans le $n^{\circ} 211$ de 1973 des AHRF, p. 112-127, Irmgard Hartig a proposé de courtes « Observations sur la querelle entre Jaurès et Mehring » où elle cherche essentiellement à défendre contre Jaurès la qualité du livre de Mehring La légende de Lessing. Elle donne à la fin de ses « observations » (p. 116-127) une traduction de l'article "Pour le roi de Prusse », différente de celle de 1903 due à Léon Rémy, mais, à la différence de ce dernier, supprime (p. 121) vingt lignes du texte original.

(13) Bruno ANTONINI, op. cit., p. 121, écrit : « Jaurès est le premier historien économique et social de la France. »

(14) Pour I'histoire de la «commission Jaurès», voir Christine PeYrard et Michel Vovelle (dir.), Héritages de la Révolution française à la lumière de Jaurès, Publications de l'Université de Provence, 2002.

(15) Le premier historien allemand à avoir, certes dans un esprit partisan anti-révolutionnaire,proposédelaRévolution,àpartirdel'étudedenombreuxdocuments, untableaud'ensemble sérieux, incluant massivement l'Europe, est en 1853 Heinrich von Sybel avec sa Geschichte der Revolutionszeit von 1789 bis 1795. Cf. Walter GRAB, «Französische Revolution und deutsche Geschichtswissen-schaft » dans Jürgen Voss (Hrsg.), Deutschland und die Französische Revolution, München und Zürich, Artemis Verlag, 1983, p. 307-308.

(16) Cf. Albert SoreL, L'Europe et la Révolution française, 7 volumes publiés de 1884 à 1904. En 1951, Godechot a critiqué cette approche trop étroitement diplomatique selon lui. Cf. Jacques GodeCHOT, "L'Europe et la Révolution française », Annales. Économies, Sociétés, Civilisations, 1951, vol. 6, n² 2, p. 214.

(17) HSRF, tome IV, p. 11. 
Ce qui intéresse Jaurès, c'est la vérité de l'Allemagne de 1789 qu'il traque en consacrant deux cent cinquante pages à ce pays avec lequel il entretient une relation particulière. D'abord, il est capable de traduire lui-même ses abondantes citations d'auteurs allemands. Ensuite, il a étudié les grands penseurs (Kant, Fichte, Hegel, Marx), ce qui lui a permis de comprendre les attentes et les susceptibilités de cette Allemagne du $\mathrm{xIX}^{\mathrm{e}}$ siècle qui, en 1870-1871, avait infligé militairement à l'orgueil de la France une si terrible humiliation. Enfin, comme socialiste, il s'intéresse à I'action militante du SPD, modèle des partis socialistes de l'époque. Dans les préoccupations de Jaurès, l'Allemagne occupe une place de choix. À son époque, il fut l'un des rares à vouloir comprendre ce pays sans a priori, ni négatifs ni positifs ${ }^{18}$.

Dans son introduction au tome IV sur La Révolution et l'Europe, Jaurès place ce volume sous le signe d'une façon « socialiste » d'écrire I'histoire " qui veut briser les étroits préjugés nationaux » ${ }^{19}$, cet ethnocentrisme français caractéristique, selon lui, d'abord des "révolutionnaires de 1792 » eux-mêmes, « qui n'avaient du reste du monde qu'une idée superficielle et vague », ensuite des historiens français de la Révolution chez qui «c'est la France presque seule qui occupe la scène. Les autres peuples sont dans une sorte de lointain ». La « conscience française » aurait " simplifiéce grand drame » en une lutte entre " deuxforces actives : la force de la France révolutionnaire et la force des tyrans coalisés; lesmultitudeseuropéennesn'apparaissentquecommeunepuissance incertaine et confuse disputée par des tendances contradictoires $»^{20}$. La réalité des pays européens serait beaucoup plus complexe que ce manichéisme ne le laisse entendre, nous dit Jaurès l'internationaliste qui voudrait guérir les Français de leur cécité face aux spécificités des cultures étrangères, de l'allemande en particulier.

L'objectif déclaré de Jaurès concernant l'Allemagne, c'est donc decomprendre son individualité et les circonstances historiques particulières qui ont empêché ce pays de faire une révolution comme la France en 1789. La question essentielleest formulée dès l'introduction : Quelle

(18) Pour plus d'informations sur la relation privilégiée de Jaurès à l'Allemagne, voir notre article: "Le refus des simplifications. L'image de l'Allemagne dans trois œuvres de Jean Jaurès: "Des premiers linéaments du socialisme allemand chez Luther, Kant, Fichte et Hegel" (1892), "La Guerre franco-allemande" (1908) et "L'Armée nouvelle" (1911) », Cahiers Jaurès, n 179 (janviermars 2006), p. 33-80. La thèse principale de Jaurès, De la réalité du monde sensible (1891), est la seule de ses cinq «œuvres » à ne pas traiter totalement ou en partie de l'Allemagne.

(19) HSRF, tome IV, p. 12.

(20) Ibid. 
est le secret de l'âme allemande ${ }^{21}$ ? Jaurès se range aux côtés de " tous ceux qui ont cherché à pénétrer le secret de l'histoire allemande ${ }^{22}$. "Âme » et histoire sont pour notre auteur, comme pour beaucoup d'historiens des civilisations jusque dans les années $1950^{23}$, des termes synonymes qui renvoient aux caractéristiques fondamentales des différents pays. Jaurès prouve une fois de plus son sens aigu de ce qu'il appelle dans l'introduction à son histoire de la Révolution « la complication presque infinie de la vie humaine $»^{24}$.

Certes, cette recherche de la vérité sur l'Allemagne de 1789 s'inscrit dansunschémacomparatistetraditionnelopposantFranceetAllemagne, mais en l'ouvrant de façon très moderne. Le comparatisme entre les deux voisins pose a priori qu'il existe une différence importante entre eux, entre, d'une part, un idéalisme allemand " profond » qui, ayant pénétréle fonctionnementdumonde,estplutôtsceptiqueetpolitiquementréservé, et, d'autre part, un idéalisme français « actif » qui veut changer le monde dès maintenant. Cette opposition a été en France codée pour des générations d'intellectuels (dont Jaurès) par $\mathrm{M}^{\mathrm{me}}$ de Staël qui, dans son livre De I'Allemagne (1810), avait souligné le pessimisme des Allemands, la hardiesse de leur pensée et de leurs idéaux et leur pusillanimité politique. Caractéristiquesquidétermineraientundédoublementdelapersonnalité allemande : "L'esprit des Allemands et leur caractère paraissent n'avoir aucune communication ensemble :I'un ne peut souffrir de bornes, I'autre sesoumetà tous lesjougs; |'un est trèsentreprenant, l'autretrès timide $»^{25}$. Cettedifférenceculturellepeutêtreexagéréeenuneopposition denature irréductible parune idéologie prônant la supériorité del'un des deux pays sur l'autre. $\mathrm{Ni} \mathrm{M}^{\text {me }}$ de Staël ni Jaurès ne pratiquent un tel comparatisme " agressif ", qui n'apparaît véritablement en France qu'après la guerre de 1870-1871 comme réaction idéologique à l'invasion allemande mais qui avaitdéjàsurgienAllemagnependantlesguerresnapoléoniennescomme réaction à l'envahisseur français. Comme le souligne Walter $\mathrm{Grab}^{26}$, ce comparatismecaractériseen particuliertoutel'historiographieallemande

(21) HSRF, p. 11.

(22) Ibid., p. 17.

(23) Cf. André Siegfried, L'âme des peuples, Paris, Hachette. Cf. aussi sur la notion d'âme notre article : Éric GUILLET, «Des profondeurs de l'âme à la chair du quotidien. Deux images de I'Allemagne en France : "L'âme allemande" (1933) de Louis ReYNAUd et "Cousins par alliance" (2002) de Béatrice Durand », Allemagne d'aujourd'hui, n 182 (octobre-décembre 2007), p. 133-149.

(24) HSRF, tome l, p. 65.

(25) $\mathrm{M}^{\text {me }}$ de STAËL, De l'Allemagne I, Paris, Garnier-Flammarion, p. 64.

(26) Walter Grab, «Französische Revolution und deutsche Geschichtswissenschaft » dans Jürgen Voss (Hrsg.), op. cit., p. 300-322. 
sur la Révolution française jusque dans les années 1950, de Heinrich von Sybel dans les années 1850 privilégiant les «valeurs » allemandes par rapport aux « valeurs » françaises issues de la Révolution, à Gerhard Ritter etWilly Andreas après 1945 . L'expérience du nazisme provoque peu à peu après 1945 un bouleversement dans l'image que les Allemands se font d'eux-mêmes. Le schéma traditionnel d'une grande différence entre France et Allemagne est conservé par les historiens en RFA et en RDA mais inversé. La théorie du Sonderweg, de la spécificité de l'histoire allemande depuis 1789,jugemaintenantnégativementcetteparticularitéhistorique en la présentant comme anormale par rapport à ce modèle d'évolution historique qu'aurait été l'histoire de la France pendant la même période. Comme l'écrit Matthias Middell : «Dans les deux États allemands, les chercheurs ont recours à l'idée de 'spécificité allemande', qui reprend la vieille tradition, tant du libéralisme allemand que du marxisme, d'un retard en comparaison de la France issue de la tradition révolutionnaire $[\ldots] »^{27}$. En RDA particulièrement, toute une série d'historiens ont étudié le « modèle »constituéparla Révolution françaiseet les mouvementsallemands apparentés à ceux des Jacobins et des Sans-culottes français ${ }^{28}$.

Dans les années 1980 s'opère dans l'historiographie allemande sur la Révolution un changement de paradigme. Le schéma dualiste centré surcequi sépare Allemagne etFrance est remis en cause par une nouvelle génération d'historiens qui donne une nouvelle vie au comparatisme en insistant au contraire sur les similitudes, les interactions, les transferts

(27) Matthias MIDDELL, «La Révolution française et l'Allemagne : du paradigme comparatiste à la recherche des transferts culturels », AHRF, n 317 (en ligne), p. 3.

(28) Parmi les recherches en RDA sur Jacobins et Sans-culottes, on citera par ordre de parution : Hedwig VoEGT, Die deutsche jakobinische Literatur und Publizistik 1789-1800, Berlin/DDR, Rütten und Loening, 1955 ; Gerhard STEINER, DerTraum vom Menschenglück, Leben und literarische Wirksamkeit von Carl Wilhelm und Henriette Frölich, Berlin/DDR, Akademie Verlag, 1959; Claus TRÄGER, Mainz zwischen Rot und Schwarz. Die Mainzer Revolution 1792/93 in Reden, Schriften und Briefen, Berlin/DDR, Rütten und Loening, 1963; Heinrich ScheEL, Süddeutsche Jakobiner, Klassenkämpfe und republikanischeBestrebungen im deutschen Süden Ende des 18. Jahrhunderts, Berlin/DDR, Akademie Verlag, 1965; Walter MARKov, Jacques Roux oder vom Elend der Biographie, Berlin/DDR, Akademie Verlag, 1966 (Markov a consacré plusieurs livres à Jacques Roux); Gerhard SteineR, Forsters Werke in zwei Bänden, Berlin und Weimar, Aufbau Verlag, 1968 (contient une excellente introduction de cinquante pages à Forster). Pour la RFA, on citera Walter GRAB (qui fut professeur à Tel Aviv) avec plusieurs livres sur le sujet dont : Eroberung oder Befreiung? Deutsche Jakobiner und die Franzosenherrschaft im Rheinland1792-1799, Trier, Schriften aus dem Karl-MarxHaus, 1971 et Leben und Werke norddeutscher Jakobiner, Stuttgart, Metzler, 1973. Pour la France, on citera les travaux de ou sous la direction de Marita GILLI : Pensée et pratique révolutionnaires à la fin du dix-huitième siècle en Allemagne, Paris, Les Belles Lettres, 1983 et « Le mouvement révolutionnaire dans les pays allemands », numéro spécial des AHRF, n 255-256 (janvier-mars, avril-juin 1984) avec un avant-propos de Marita Gilli. 
culturels. Apparaissent des « analyses plus fines qui mettent en avant les interactions plus que la construction des différences ${ }^{29}$. Rolf Reichardt pose la question de savoir si la Révolution française peut servir de référence pour mesurer la spécificité historique allemande ${ }^{30}$. Il redonne en quelque sorte sa normalité à l'histoire allemande en soulignant les ressemblances en 1789 entre France et Allemagne et en déplaçant les différences sur le plan des mentalités, de la perception différente des mêmes événementsdanslesdeuxcultures.En 1985, cette« perspectiveculturelle prend alors la place d'une interprétation de la Révolution principalement politique et sociale $»^{31}$. Cette nouvelle perspective basée sur l'étude de la communication entre les deux cultures a la particularité d'être foncièrement ouverte, à la fois internationale et inter-disciplinaire ${ }^{32}$. Toutefois, comme Middell le souligne bien, elle ne s'oppose pas radicalement au vieux comparatisme dualiste mais ne vise qu'à en corriger les excès, à le compléter, à l'affiner ${ }^{33}$. Cet abandon de la théorie du Sonderweg va d'ailleurs de pair avec l'abandon de celle de l'unicité de la Révolution française et peut conduire à affirmer soit, comme Annie Jourdan ${ }^{34}$, qu'il y a eu à la fin du xvIII siècle des révolutions ailleurs qu'en France, soit, comme Franz Dumont, que la norme dans le monde était alors plutôt l'absence de révolution ${ }^{35}$.

D'un comparatisme fermé qui « présuppose des aires culturelles closes ${ }^{36}$ à un comparatisme ouvert, telle est l'évolution qu'a connue, sur le sujet « France et Allemagne en 1789 », I'historiographie allemande et internationale. Or le comparatisme pratiqué par Jaurès est profondémentmodernepuisqu'il supposequeles airesculturellesfrançaiseetallemandesontfoncièrementouvertesl'uneàl'autre. Fidèleà son principede l'unité de l'être ${ }^{37}$, il pense que, à condition que les hommes le veuillent, les forces centripètes d'union finiront toujours par l'emporter sur les forces

(29) Matthias MidDELL, op. cit., p. 4.

(30) Rolf REICHARDT, "Die Französische Revolution als Maßstab des deutschen Sonderweges? Kleines Nachwort zu einer großen Debatte » dans Jürgen Voss, op. cit., p. 323.

(31) Matthias MIDDELL, op. cit., p. 5.

(32) Ibid.

(33) Ibid., p. 16.

(34) Annie Jourdan, La Révolution, une exception française?, Paris, Flammarion, 2004.

(35) Franz Dumont, « Mainz und die Französische Revolution » dans Jürgen Voss, op.cit., p. 140 : « D'ailleurs, il semble erroné de demander pourquoi, vers 1800, à Mayence comme dans toute l'Allemagne, il n'y a pas eu de révolution. Car ainsi l'évolution française est érigée en référence unique bien que pourtant, dans l'ensemble, elle représente plutôt l'exception ». (traduit par nous)

(36) Michel Espagne, «Sur les limites du comparatisme en histoire culturelle », Genèses $\mathrm{n}^{\circ} 17,1994$, p. 115.

(37) Cf. notre article, op. cit., p. 34-37. 
opposées centrifuges de division. Il voit toujours les différences entre la France et l'Allemagne sur un fond de ressemblances essentielles.Comparatisme ouvert donc, qui croit cependant globalement à la supériorité du modèle révolutionnaire français etn'exclutpas quecertaines conclusions de Jaurès doivent, sur fond de la recherche actuelle, être relativisées.

Points communs entre l'Allemagne et la France en 1789

Premier point commun : penseurs allemands et français partageaient les mêmes idéaux de justice et de fraternité entre les hommes et considéraient la société autour d'eux d'un œil également sévère. Les " esprits » allemands auraient en effet été marqués par l'esprit critique caractérisant la pensée française des Lumières ${ }^{38}$. Les penseurs français auraient transmis à leurs collègues allemands « la passion de la science et de l'humanité $"$ ", auraient éveillé en eux « le besoin de tout comprendre et de tout harmoniser $»^{40}$. Jaurès suppose ici, sans en préciser les modalités, un vaste transfert culturel de la France des Lumières vers I'Allemagne de l'Aufklärung, transfert à sens unique qui suppose la supériorité d'un modèle français rayonnant sur l'Allemagne. Rudolf Vierhaus reconnaît luiaussila présence diffused'attentes radicales dans la sociétéallemande de 1789 mais nie par contre qu'elles aient été « importées » de France ${ }^{41}$.

Second point commun : I'attachement des Allemands à leur pays n'est en 1789 pas moins fort que celui des Français, même si l'Allemagne est unenébuleuse de plusieurs centaines d'États. Nepouvant être fiers de la faiblesse politiqued'un tel manteau d'Arlequin, les patriotes allemands s'identifient surtout à leur culture, à leurs écrivains et à leurs penseurs. Et c'est une grave erreur des révolutionnaires français, selon Jaurès, d'avoir étéaveuglesaux « terriblessusceptibilitésnationalesd'un peupled'autant plus fier et ombrageuxqu'il ressentait douloureusement la contradiction de sa force interne et de sa destinée $»^{42}$.Ce puissant patriotismeallemand sera, à partir de 1792, la raison principale de l'échec des révolutionnaires français dans leur tentative d'exporter la révolution en Allemagne puisque, de toutes les forces qui travaillent la société allemande à la fin du xvIII ${ }^{e}$ siècle, c'est celle qui, sous l'occupation napoléonienne, finira

(38) HSRF, tome IV, p. 13.

(39) Ibid., p. 14.

(40) Ibid.

(41) Cf. Rudolf Vierhaus, "Sie und nicht wir" Deutsche Urteile über den Ausbruch der Französischen Revolution » dans Jürgen Voss, op. cit., p. 15.

(42) HSRF, p. 24. 
parl'emporter, donnantuneteintenationale, puis nationalisteauxaspirations radicales allemandes à plus de vérité et de justice. À la fin du tome IV, Jaurès souligne cet appauvrissement, selon lui, du patriotisme révolutionnaire : "Ce n'est que peu à peu, et sous une forme nationaliste, que I'Allemagne assimilera une partie de la Révolution française $»^{43}$, prélude à un long antagonisme franco-allemand.

Jaurèsconsacrelechapitretrois « L'expansion révolutionnairefrançaise » (quinze pages) à ce drame des révolutionnaires français. Dans les pays occupés, ils avaient voulu, par le décret du 19 novembre 1792 accordant « fraternité et secours à tous les peuples qui voudront recouvrer leur liberté », aider les Européens à se libérer plus rapidement de l'Ancien Régime mais ne contribuèrent finalement qu'à développer un nationalisme anti-français de plus en plus réactionnaire, surtout après le décret du 15 décembre 1792qui instituaitl'administration révolutionnaire dans les pays conquis ${ }^{44}$. Jaurès déplore : «La liberté prenait la forme et les mœurs de la conquête $»^{45}$. C'est ainsi, nous dit-il, que même de jeunes étudiants allemands, comme Hegel et Schelling, d'abord tout acquis à la Révolution, se détournèrent d'elle fin $1792^{46}$.

Concernant ce patriotisme, qui jouera sous l'occupation napoléonienne un rôle si essentiel dans le refus final de la Révolution par I'Allemagne, Jaurès pense que les succès militaires de Frédéric II de Prusseetl'amplevolontéréformatrice de Joseph Ilen Autricheont contribué à convaincre beaucoup d'Allemands de la fin prochaine de l'impuissance politique de leur nation et ainsi renforcé leur fierté nationale ${ }^{47}$. Frédéric II particulièrement « était pour toutes les classes du peuple allemand, pour les soldats comme pour les lettrés, pour les paysans comme pour les artistes, le héros de la renaissance nationale $»^{48}$. Il aurait fasciné les Allemands, cultivés ou non, par la façon dont il avait osé s'émanciper de la tutelle française en affirmant les intérêts de la Prusse sans craindre de provoquer la France. Jaurès croit donc à « l'influence de Frédéric II sur la grande pensée allemande ${ }^{49}$, c'est-à-dire sur la pensée allemande des Lumières, dans la mesure où « il avait délivré l'Allemagne des chaînes de

\author{
(43) Ibid, p. 255. \\ (44) Ibid., p. 147-151. \\ (45) Ibid., p. 146. \\ (46) Ibid., p. 144. \\ (47) Ibid., p. 48. \\ (48) Ibid., p. 49. \\ (49) Ibid., p. 52.
}


l'imitation et de la peur $\aleph^{50}$. Cette conception oppose Jaurès au marxiste Franz Mehring, qui pense, comme il l'expose dans La légende de Lessing, que les « despotes éclairés » allemands n'ont eu aucune influence positive sur les penseurs des Lumières comme Lessing ou Herder qui les ont souvent critiqués. La thèse d'une « pénétration de l'action royale et de la pensée allemande $»^{51}$ par le biais du patriotisme, telle que la reprend ici Jaurès, serait une légende. Jaurès polémique d'ailleurs ici nommément avec Mehring : « Pourquoi, en se refusant à voir l'action éclatante et fascinatrice de Frédéric II, Mehring se condamne-t-il par là même à ne pas comprendre l'histoire de l'Allemagne moderne $~^{52}$ ? La polémique entre JaurèsetMehringnoussemblereposersurunmalentendu.Mehring considère surtout que Frédéric II a fait peu de cas de la littérature allemande contemporaine s'écartant sciemment du goût français classique et que beaucoup d'écrivains allemands n'ont guère apprécié son despotisme, tout éclairé qu'il ait pu être. Jaurès ne nie pas ce fait historique mais considère seulement que les victoires du roi de Prusse ont eu un effet psychologiqueenredonnantconfianceàune « nationallemandeanéantie par la Guerre de Trente Ans ». La recherche allemande actuelle confirme d'ailleurs en partie, pour l'Allemagne protestante, l'analyse de Jaurès. Dans sa récente biographie remarquée sur Frédéric II, Johannes Kunisch nous dit que la Guerre de Sept Ans « fit apparaître dans les territoires protestants de l'Empire une conscience patriotique et se développer un sentimentidentitairequicommençaàsupprimerladistanceentrepouvoir et sujets caractéristique de l'époque de l'absolutisme $»^{53}$.

Troisième point commun auquel Jaurès consacre la plus grande partie du premier chapitre «La condition politique et économique de I'Allemagne » (cinquante pages): France et Allemagne auraient connu un développement économique structurellement similaire reposant sur l'essordu capitalismeindustriel manufacturier ${ }^{54}$.De« toutecettepoussée capitaliste, toutce travail obscurou éclatant de transformation $»^{55}$, de « ce magnifique épanouissement des industries libres ${ }^{56}$, Jaurès trouve les preuves dans les témoignages de deux écrivains allemands de l'époque Justus Möser et Georg Forster qu'il cite abondamment. Il pense « qu'il

(50) Ibid., p. 51.

(51) Ibid., p. 49.

(52) Ibid.

(53) Johannes Kunisch, Friedrich der Große. Der König und seine Zeit, München, Beck, 2005 ( $5^{\mathrm{e}}$ édition), p. 439 (traduit par nous).

(54) HSRF, p. 35.

(55) Ibid.

(56) Ibid., p. 38. 
n'y a pas pleine stagnation et routine: I'Allemagne industrielle, sans avoir l'essor de la France, est dans une crise de transformation qui atteste la puissance de forces jeunes ${ }^{57}$. Jaurès reconnaît certes quel'Allemagne n'avait pas atteint en 1789 le niveau économique de la France mais, s'il insiste sur le fait que les structures du développement étaient les mêmes danslesdeuxpays,c'estpoursoulignercombienlesexplicationspurement économiquesdeschangementssociauxsontinsuffisantes.Comme,économiquement, I'Allemagne était presque au même niveau que la France, une révolution aurait, selon les lois historiques du « matérialisme économique ", dû éclater aussi en Allemagne. Or tel ne fut pas le cas, preuve, selon lui, que d'autres raisons, « les forces d'ordre politique et intellectuel ${ }^{58}$, se sont combinées aux forces économiques pour provoquer la révolution en France et l'empêcher outre-Rhin : « Isolé, le mouvement économique n'est qu'une abstraction $»^{59}$. Jaurès dénie au « matérialisme économique »sa prétentionà expliquer les mouvements historiques : ॥I ouvredesjours nouveauxsurla profondeurdes phénomèneshistoriques, mais il n'en épuise pas la réalité $»^{60}$. Paroles fortes par lesquelles Jaurès prend sciemment le contre-pied de l'interprétation marxiste de l'histoire allemande comme déterminée par les seules forces économiques, c'està-dire par l'absence d'une bourgeoisie capitaliste dynamique. Jaurès va même jusqu'à soupçonner les marxistes d'avoir falsifié l'histoire pour mieux mettre en valeur leur thèse du primat des forces économiques sur les forces politiques et intellectuelles ${ }^{61}$.

L'affirmation selon laquelle l'économie allemande en 1789 aurait été déjà bien développée, certes moins que l'économie française, est corroborée par Hans-Ulrich Wehler qui écrit : «L'Allemagne était reliée de multiplesfaçonsaudynamismedusystèmeéconomiqueoccidental, bien qu'elle ne fît pas encore partie des très grands centres de son développement $»^{62}$. Ce développement économique de l'Allemagne, Jaurès le mesure exclusivement à l'importance des manufactures, influencé qu'il est par Marx qui, dans le douzième chapitre du Capital, surestime cellesci en en faisant le stade de concentration industrielle ayant chronologiquement précédé l'usine. Or dès les années 1920, comme le souligne

(57) Ibid, p. 35.

(58) Ibid., p. 40.

(59) Ibid.

(60) Ibid.

(61) Ibid.

(62) Hans-Ulrich WeHLER dans Deutsche Gesellschaftsgeschichte Erster Band (1700-1815), München, Beck, 1989, p. 123 (traduit par nous). 
Wehler ${ }^{63}$, Werner Sombart et MaxWeber contestent cette thèse marxiste. Selon eux, manufactures, usines et système, traditionnel en Allemagne, du Verlag (fabrication de produits à domicile pour de grands marchandsfabricants ${ }^{64}$ ) auraient coexisté. Quant à la thèse selon laquelle ce serait la bourgeoisie industrielle qui aurait été l'instigatrice de la Révolution en France et aurait donc pu l'être aussi, en toute logique, en Allemagne, elle est battue en brèche par Reichardt :

"Or la recherche actuelle a établi que les Lumières françaises aussi bien que la Révolution n'ont pas été portées par une bourgeoisie industrielle à tendance "capitaliste" (elle restait plutôt le plus possible en dehors de la politique), mais par une "bourgeoisie d'Ancien Régime" [en français dans le texte] impliquée de multiplesfaçons dans les vieilles institutionsd'État, principalementpardesbourgeoisrentierspassifséconomiquementetdesjuristes détenteurs dechargesainsique, untemps, parune minorité influente de nobles libéraux ${ }^{65}$.

En accentuant les similitudes entre Allemagne et France, Jaurès se montre un adepte de l'interprétation que le révolutionnaire Barnave avait proposéedel'histoireeuropéennedanssonlivreposthume :Introductionà la Révolution française ${ }^{66}$. Il estimait que celle-ci n'était pas un événement isolé, ni dans le temps ni dans l'espace, mais l'expression, certes unique danssabrutalité,d'uneatmosphèreeuropéennerévolutionnairecaractérisée principalement par une évolution des rapports de propriété au profit de la bourgeoisieetdes(gros)paysansentraînantnécessairementàplusoumoins brève échéance, selon les pays, un déplacement du pouvoir politique.

Jaurès aimerait comprendre pourquoi le radicalisme diffus dans toute l'Europe en 1789 n'a abouti qu'en France à une éruption violente. À cette fin, il met en lumière les éléments particuliers à l'Allemagne qui ont empêché les éléments communs de se combiner, comme en France, pour libérer leur important potentiel révolutionnaire. Trois de ces causes particulières sont d'ordre politique : le morcellement du territoire en une poussière de principautés, la dépendanceéconomiquedela bourgeoisie par rapport à ces nombreuses cours princières et le despotisme éclairé. Les deux autres causes sont d'ordre intellectuel : le primat, chez les penseurs, de l'évolution sur la révolution et la tendance au pessimisme.

(63) Ibid., p. 103

(64) Cf. Michel HAU, Histoire économique de l'Allemagne $\mathrm{XIX}^{\mathrm{e}}-\mathrm{xx}$ e siècle, Paris, Economica, 1994, p. 5-7.

(65) Rolf ReICHARDT, op. cit., p. 326 (traduit par nous).

(66) BaRnave, Introduction à la Révolution française, texte présenté par Fernand Rude, Paris, Colin (Cahiers des Annales), 1960. 
Spécificités du radicalisme allemand en 1789

Ces cinq facteurs auraient concouru à affaiblir bourgeois et « prolétaires » allemands, la bourgeoisie en particulier qui profitait certes, comme la bourgeoisie française, de l'essor industriel mais, à la différence decelle-ci,manquaitd'assurance, soumisequ'elleétaitauxinnombrables princes : " La bourgeoisie allemande, en cette fin du xvIII siècle, existait à peine; ou du moins elle n'avait pas cette confiance en soi que donnent la croissance de la richesse et l'essor des entreprises ${ }^{67}$.

Ce manque d'aplomb de la bourgeoisie allemande aurait d'abord son origine dans la multitude d'États particuliers auxquels « des liens multiples d'habitude, d'intérêtet de vanitéattachaientencore bourgeois et prolétaires ${ }^{68}$. Ce morcellement politique attisait de "violentes rivalités provinciales », favorisait le particularisme et " empêchait les mouvements d'ensemble $»^{69}$. La lutte contre les princes, les nobles et le clergé ne trouvaient pas en Allemagne, comme en France, d'espace politique assez vaste pour concentrer son énergie : « Dans la France centralisée et à peu près unifiée, même avant 1789 , le terrain large et uni se prêtait, si l'on peut dire, à des opérations de masses $»^{70}$. Bourgeois et prolétaires français, à la différence de leurs congénères allemands, « disposaient de toute leur énergie contre les privilèges des nobles et du clergé, contre l'arbitraire des rois et des bureaux ${ }^{{ }^{71}}$.

Cet émiettement donnait à la myriade des princes un poids particulier sur le plan politique et sur le plan économique, puisque, nous dit Jaurès, labourgeoisieétaitfortementtributairedesbesoinsdescoursallemandes $^{72}$.Ledynamismeindustrieldelabourgeoisieétaitpresqueauseul service de la noblesse et du clergé. Tel n'était pas le cas en France où elle constituait, à côté de l'aristocratie, un important débouché pour ses propres produits. En Allemagne, pareille dépendance économique visà-vis des deux premiersétats s'accompagnaitnécessairementd'unegrande docilité politique. Jaurès n'hésite pas à parler dans son langage percutant à la fois imagé et caustiqued'un « peupled'antichambreet decathédrale, habitué à des dorures d'église et de domesticité $»^{73}$.

Cettepropensiondesbourgeoisallemandsàsemontrerbonssujets aétérenforcée, ajouteJaurès, parlephénomènedudespotismeéclairéqui

\footnotetext{
(67) HSRF, tome IV, p. 17.

(68) Ibid., p. 15.

(69) Ibid.

(70) Ibid.

(71) Ibid.

(72) Ibid., p. 41.

(73) Ibid., p. 187.
} 
satisfaisait à la fois le radicalisme des Allemands et leur habitude d'obéissance aux souverains. Joseph II de Habsbourg et Frédéric II de Prusse avaienteneffetessayéderévolutionnerleursroyaumesparenhaut,imbriquant ainsi l'ancien et le nouveau : "Ils avaient habitué l'Allemagne à concevoir le progrès non comme l'effort collectif et libre de la nation, mais commeunacted'autorité ${ }^{74}$. Les deuxgrands despoteséclairésgermaniquesauraientainsi, parleurvolontédechangementparenhaut,justifiéla traditionnelle soumission de leurs sujets et contribuéà la pérenniser en prouvant par leurs réformes l'inutilité d'une révolution par en bas.

À ces trois raisons politiques du manque de pugnacité de la bourgeoisieallemande, Jaurès en ajoute deuxautres qui relèvent du domaine des mentalités : la conviction des penseurs allemands que des changements profonds ne peuvent qu'être le résultat de lentes évolutions intérieures et leur pessimisme.

À la veille de la Révolution, bien des penseurs allemands aspirent à un bouleversementen profondeur des comportements humainsquine peut qu'être le fruit d'un long travail d' "éducation du genre humain ", titre d'un livre de Lessing que Jaurès analyse ${ }^{75}$. Pareil changement radical ne peut se faire qu'extrêmement lentement. C'est une évidence pour les penseurs allemands qui incluent nécessairement le long terme dans leur réflexion sur une société idéale. Cela signifie concrètement qu'ils répugnent à une action politique immédiate comme la Révolution française dontils ne retiendront finalementquela brutalitéet la superficialité. La pratique révolutionnaire s'avérera décevante pour des gens rêvant de concorde et de révolution des mentalités plus que de liberté civile. HardiessethéoriqueetpusillanimitépratiqueconstituentpourJaurèslestraits principaux de la penséeallemande en $1789^{76}$. Pareille prioritédonnéepar les grands penseurs allemands à l'évolution sur la révolution aurait freiné l'ardeur révolutionnaire de la bourgeoisie allemande.

Unautrefaitdementalitéauraitempêchécettebourgeoisiedepasser à l'action : le pessimisme des penseurs allemands qui doutaient des chances de succès de l'action politique immédiate. Ils avaient en effet vu les réformes de Joseph II se briser contre la résistance violente du clergé et de la noblesse : " Ainsi l'impuissance constatée du despotisme éclairé à ouvrir des voies nouvelles, à accomplir des réformes, laissait dans la pensée de l'Allemagne un doute pesant et triste $»^{77}$. Les Lettres pour

(74) Ibid., p. 64.

(75) Ibid., p. 91-92.

(76) Ibid., p. 64.

(77) Ibid., p. 56. 
I'humanité de Herder par exemple seraient imprégnées de « cette sorte de tristesse universelle et de déception ${ }^{78}$. Même des révolutionnaires convaincus comme Forster auraient déduit de l'échec des réformes de Joseph II « que le progrès est une œuvre difficile et lente, qu'il est impossible de brusquer. Ainsi l'activité réformatrice de l'empereur se tourne en uneleçondepatience, derésignation, detemporisation $»^{79}$.Sensdeslentes évolutions intérieures et pessimisme inhibant sont les deux faces d'une même attitude : «Attendons, sous la succession lente des tièdes soleils, l'incertaine maturation des semences cachées $»^{80}$.

Une telle culture, qui exige beaucoup du monde mais craint de se salir les mains, aurait, selon Jaurès, ses racines dans la Réforme luthérienne. D'abord dans la pensée même de Luther : «Il croyait avoir renoué le vrai mouvement de la pensée chrétienne, et c'est à l'intérieur même et au plus profond du christianisme qu'il avait éveillé la liberté moderne de penser ${ }^{81}$. Ensuite, dans les violentes guerres intestines, sources de maux indicibles, que la Réforme a entraînées et qui ont déprécié l'action dans l'esprit de bien des Allemands ${ }^{82}$.

Cescinqspécificitésconflueraientdansuneaversiongénéralepour l'action politique directe, immédiate, violente. Alors que le tiers état françaisn'a, en 1789, plus de patienceavecsesclasses dirigeantes et laissebrutalement exploser son désir de libération, I'Allemagne, malgré une aussi forte aspiration à la liberté se manifestant dans un enthousiasme initial assez général pour la Révolution française, préfère rester politiquement soumiseà son clergé et à sa noblesse. Â côté des ardents révolutionnaires français, les bons sujets allemands semblent bien pâles, effacés, craintifs, rêvantcertesàl'avènementd'un mondemeilleurmaisseulementdansun avenir lointain et incertain. Jaurès est frappé par « l'hésitante conscience révolutionnaire de l'Allemagne $»^{83}$, par son « indécision générale ${ }^{84}$, par son « état étrange et ambigu »" Cette différence fondamentale entre les deux pays, ill'exprime par la métaphore dela lune allemande et du volcan français : « Aucun souffle chaud de Révolution n'est passé sur la bourgeoisie allemande. [...] C'est une lune rêveuse et pâle qui se lève derrière la

\footnotetext{
(78) Ibid.

(79) Ibid., p. 58.

(80) lbid., p. 60.

(81) Ibid., p. 64.

(82) Ibid.

(83) Ibid., p. 60.

(84) Ibid., p. 120.

(85) Ibid., p. 130.
} 
cime empourprée du volcan $»^{86}$. Jaurès évoque en Allemagne " ces flambeaux d'attente, pâlis par la lumière brutale du matin ${ }^{87}$.

Jaurès utilise également une métaphore géométrique, celle de la ligne courbe, symbole de la pensée allemande sachant que la réalisation de l'idéal peut emprunter des voies compliquées et paradoxales, et de la ligne droite, symbole de cette France révolutionnaire voulant immédiatement la satisfaction de revendications concrètes : «C'est dans des courbes, des replis et des enveloppements sans fin que l'esprit allemand se meut vers son but sublime, qui est l'assimilation de l'univers par la penséesouveraine.Maiscommecettegéométriedescourbesestpeufavorable à l'élan direct des révolutions ${ }^{88}$ !

La «pensée impuissante » des grands penseurs allemands

Danslelong deuxièmechapitre « Lapenséeallemande »(p. 69-144), Jaurès illustre cette thèse d'une culture allemande qui peine à sortir du « cercle de la passion impuissante ${ }^{89}$ en citant les grands penseurs de l'époque: Goethe, Herder, Kant, Lessing, Schiller, Wieland. Et Jaurès d'osciller entre une grande admiration pour les idéaux proclamés dans leurs livres par tous ces esprits supérieurs et le regret qu'ils aient tous répugné à passer à l'action politique ${ }^{90}$.

On ne considérera ici que les propos de Jaurès sur Kant et Schiller. Kant représente en effet emblématiquement pour Jaurès la « méthode allemande », influencée par « la politique de Frédéric II, accordant toute la liberté aux opinions, au moins en matière religieuse, et instituant partout une exacte discipline ${ }^{91}$. Cette $"$ dualité » consiste à « dissocier la conduite pratique et la vie idéale de l'esprit $»^{92}$, I'usage public de la raison, tenue de se conformer docilement aux lois de la société du moment, et son usage privé pouvant donner libre cours à des pensées révolutionnaires : «Kant se préoccupe tout à la fois d'assurer la liberté absolue de la science et de ménager le mécanisme gouvernemental et administratif prussien $»^{93}$. Pour Kant, il ne s'agit nullement d'un sophisme car, pensant sur le long terme, il compte que le développement de la liberté de penser finira par faire éclater la gangue de toutes les règles coercitives :

(86) Ibid., p. 48.

(87) Ibid.

(88) Ibid., p. 92.

(89) Ibid., p. 245.

(90) Ibid., p. 69.

(91) Ibid., p. 103.

(92) Ibid., p. 103.

(93) Ibid., p. 102. 
« II ne ruse pas avec le droit de la pensée libre. II faut qu'elle ait toujourslecouragedes'affirmer.Etcettepenséelibre, en sepropageant, refoulera les préjugés et réformera les institutions. Mais ce sera une évolution intérieureetlente. Les révolutions, celles quichangent seulement laforme du pouvoir, ne sont que des accidents superficiels et sans valeur. C'est du dedans au dehors que les vraies révolutions doivent s'accomplir $»^{94}$.

Kant prétend même que plus la contrainte extérieure exercée par les institutions est forte, plus le besoin de liberté augmente. Il pense de manière générale que, plus se déchaînera l'impitoyable animalité de l'homme, telle que cupidité, ambition, orgueil et inquiétude la manifestent, plusl'homme s'épuisera et finira par reconnaître les avantages de la concorde et de la modération : « L'éducation de l'humanité se fera par la culture intérieure et la réflexion, elle se fera aussi par la douleur $»^{95}$. En tant que Français persuadé des vertus, dans certaines circonstances historiques, de l'action révolutionnaire, Jaurès est certes un peu surpris par cette pensée « à la fois mâle et réservée, vigoureuse et prudente ${ }^{96}$ mais, en mêmetemps,iladmireprofondémentcet « optimismeréalistede Kant » qui, « s'il est rebelle aux impatiences et aux fièvres, est prémuni contre tout désespoir ou même contre toute lassitude ${ }^{97}$. Ayant d'abord approuvé la Révolution pour sa tentative d'organiser rationnellement l'État, Kant s'en détournera lorsqu'elle deviendra violente.

Pour les mêmes raisons, Jaurès admire Schiller auquel il consacre aussi de nombreuses pages. Certes, il lui reproche le côté vague et un peu naïf de son idéal d'une humanité meilleure tel qu'il s'exprime dans le fameux Hymne à la joie : «Il attend l'émancipation du monde beaucoup moinsd'unactedevolontédesclassesasserviesqued'une sortededouce et universelle floraison de bonté ${ }^{98}$. L'action révolutionnaire ne ferait pas partie de l'horizon de Schiller, même du jeune rebelle s'insurgeant littérairement contre la société de son temps dans sa pièce Les brigands, « un cri de désespoir plus qu'un appel à l'action ${ }^{99}$. Certes aussi, Jaurès a du mal à comprendre que le grand écrivain se soit dès le début défié de la Révolution dont les violences prouvèrent rapidement au kantien qu'il était que l'homme, n'ayant pas encore dompté l'animal en lui, n'était pas apte à faire un usage rationnel de la liberté civile fraîchement conquise : «Défiance d'idéaliste qui a peur que son rêve trop haut et trop beau ne 
soit déformé et abaissé par les faits $»^{100}$. Jaurès reproche à Schiller d'avoir, du haut de son rêve, été "sévère », « injuste pour ceux qu'aveugle dans la triste vallée la poussière sanglante de la bataille ${ }^{101}$. Pourtant, Jaurès trouve admirable « cette sérénité clairvoyante et sévère » qui fait écho à « l'optimisme réaliste » de Kant :

"Schiller ne désespère pas de l'humanité; il croit au contraire avec certitude et il sait que par l'éducation elle se libérera. Pas d'illusion sur le présent; mais aucun fléchissement de l'espérance. Le grand poète était injuste pour ses contemporains et pour la France révolutionnaire. [...] Mais quelle pénétration et quelle profondeur du regard $»^{102}$ !

Dans cet hommage lucide à Kant et à Schiller, Jaurès se situe sciemmentauconfluentdedeuximportantestraditionseuropéennesqu'ilessaie de concilier dans sa pratique d'historien et d'homme politique : celle de la grande pensée allemande " évolutionniste » et celle de l'action révolutionnaire française.

Deux « hybrides » franco-allemands : Forster et Fichte

Ce que Jaurès reproche aux penseurs allemands, c'est leur indécision viscérale à un moment clé de l'histoire universelle. Il y eut pourtant, pour confirmer la règle, des exceptions comme Forster et Fichte ${ }^{103}$.

Le plus connu des révolutionnaires allemands convertis à l'activismefrançaisestGeorgForster ${ }^{104}$,remarquablereprésentantdes Lumières célèbre à la fin du xvIII ${ }^{e}$ siècle dans toute l'Europe pour avoir accompagné Cook dans son voyage autour du monde de 1772 à 1775 dont il a publié une relation que Jaurès qualifie de « admirable, $d$ 'une netteté d'idées et d'images, d'une force et d'une rapidité de style que l'Allemagne n'avait pas encore connues » ${ }^{105}$. Jaurès dédie tout le chapitre IV « Les Allemands de la rive gauche du Rhin » (cinquante pages) au drame de ce penseur atypique en ce qu'il a choisi, à la différence de ses confrères, de se salir les mains pour essayer de réaliser l'idéal humaniste d'un Kant ou d'un Schiller. Jaurès souligne combien, certes, Forster était bien allemand, par

(100) Ibid., p. 131.

(101) Ibid., p. 134-135.

(102) Ibid., p. 135.

(103) Ibid., p. 212.

(104) Sur Forster, cf. Marita GıLı, Un révolutionnaire allemand, Georg Forster (1754-1794), Paris, Éditions du CTHS, 2005. L'ouvrage contient une introduction de Marita Gilli au penseur et la traduction de certains de ses écrits, dont les grands discours tenus au club de Mayence. Cf. aussi Marita GILL, « La conception de la révolution d'un révolutionnaire allemand : Georg Forster. Rupture ou reconstruction? », AHRF, n 347, janvier-mars 2007, p. 67-81.

(105) HSRF, tome IV, p. 165. 
exempleparson pessimismequantà la possibilitéd'amenderl'humanité. Au cours de son voyage autour du monde, il est souvent pris de dégoût devantlecomportementdesEuropéensetdesindigènes : L'humanitéest encore atroce et vile, cruelle, lubrique, avide ${ }^{106}$. Lorsque la Révolution éclate, il s'enthousiasme aussitôt pour elle, mais reste sur ses gardes ${ }^{107}$. II ne se fait guère d'illusion sur la possibilité de secouer la « langueur », la « médiocrité somnolente », la « morne immobilité » de l'Allemagne politique $^{108}$.Sonscepticismenaturelestrenforcéencoreparleregardduscientifique ${ }^{109}$. Comme les autres penseurs allemands, il tend donc à penser qu'il faut attendre des circonstances plus favorables et miser sur le long terme de l'éducation progressive du genre humain ${ }^{110}$. Mais rapidement, Forster, à la différence des autres grands penseursallemands, domine son attentisme. Lorsque les armées françaises du général Custine occupent après Valmy la rive gauche du Rhin à l'automne 1792 et y abrogent le système féodal, il est à Mayence l'un des fondateurs, sur le modèle du club des Jacobins, de la «Société des Amis du peuple » qui demandera rapidement à la Convention le rattachement à la France de la rive gauche duRhin. Isolés, soutenus ouvertement par une infime partie dela population, Forster et ses amis devront s'exiler.

Fichte est un autre penseurallemand dont les idées politiques radicales sont très marquées par la Révolution. Jaurès a déjà consacréau penseur politique de nombreuses pages dans sa thèse en latin de 1892 Des premiers linéaments du socialisme allemand où il le présente comme un des pionniers du collectivisme marxiste ${ }^{111}$. À présent, il réserve presque tout le cinquième chapitre " Fichte et la Révolution française » (une trentaine de pages) à ce penseur "socialiste " $^{112}$ dont il analyse les idées profondément révolutionnaires sur le servage, la propriété, la force de travail, le régime seigneurial et les biens d'Église. En 1793, Fichte a pris sans réserve parti contre les princes pour la Révolution, surtout dans ce que Jaurès appelle « un livre admirable : Rectification des jugements du public sur la Révolution française $»^{113}$ qu'il cite et commente abondamment. Fichte y dénonce tous les privilèges féodaux et cléricaux et n'a aucune pitiépourles privilégiés expropriés. Son analyseéconomiquedes

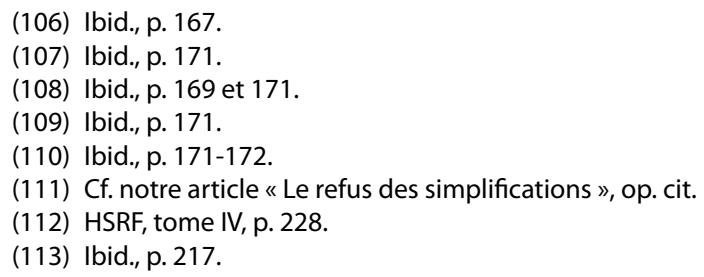


rapports sociaux est aussi, selon Jaurès, " plus profonde »"14 que celle des révolutionnaires français, de Saint-Just par exemple, puisqu'il défend " l'idée que toutes les institutions économiques et sociales sont, au fond, un "contrat de travail", implicite ou explicite, dans lequel la force de travail de l'homme est engagée $~^{115}$. Pour Fichte, la Révolution française n'est qu'une « forme nouvelle de l'éternel contrat de travail qui toujours en son fonds avait impliqué le droit de la personne humaine à disposer de soi » ${ }^{116}$. Mais Jaurès souligne que cette pensée hardie, mélange remarquable de la profondeur d'un Kant et de l'activisme des révolutionnaires français, resterait profondément allemande, c'est-à-dire craintive, lorsqu'il s'agit de passer à l'action concrète. Dans le cas de l'abolition des charges féodales, il préconise par exemple que les nobles en soient indemnisés. Jaurès ne comprend pas « ce souci des ménagements et des transitions ${ }^{117}$, pas plus qu'il ne comprend que Fichte accepte que l'État exproprie les biens d'Église seulement si les individus déclarent tous sans exception se séparer de l'Église. Unanimité jugée totalement « chimérique " par Jaurès qui préfère la solution, certes brutale, mais seule viable, choisie par les révolutionnaires français : séculariser d'un coup tous les biens d'Église. Fichte ignorerait les «nécessités de l'action » ${ }^{118}$, ce qui fait que la solution proposée par lui pour régler le problème des biens d'Église serait à la fois plus hardie en théorie et plus timide en pratique que celle des légistes révolutionnaires français :

"Elle est plus hardie en ce qu'elle fait de la revendication des biens d'Église l'affirmation suprême de la conscience libérée. [...] C'est dans la profondeur de la conscience que ce contrat illusoire se dénoue, comme il s'y était noué. [...] C'est en un drame intime et profond de la conscience et delapensée,c'estenunesortedetragédieintérieurequeserésoutpour Fichte la grande expropriation révolutionnaire des biens d'Église $»^{119}$.

Comme tous les penseurs allemands, Fichte attendrait la grande libération de l'humanité non pas d'une superficielle révolution juridique mais d'une grande révolution des mentalités qui ne peut que résulter de "l'éducation intérieure des consciences ${ }^{120}$ et de l'action persévérante de souverains éclairés. Et Jaurès d'opposer l'idéalisme actif et modeste

(114) Ibid., p. 227.

(115) Ibid.

(116) Ibid., p. 222.

(117) Ibid., p. 231.

(118) Ibid., p. 241.

(119) Ibid.

(120) Ibid., p. 218. 
des Français et l'idéalisme profond et exigeant des Allemands. En arrachant à l'Église ses biens, les révolutionnaires français se sont contentés de percer dans la « vieille forêt de préjugés et d'erreurs » entretenue par elle quelques "routes toutes droites», juste "pour que la Révolution passât. Bien des murmures, des croyances et des rêves d'autrefois continuaient à flotter dans la vieille forêt humaine. Qu'importe! La trouée de la Révolution était faite ». Un révolutionnaire allemand comme « Fichte, au contraire, avant denationaliseret deséculariserlaterre, demandaitaux arbres et aux brins d'herbe de renoncer aux flottantes chansons de jadis, aux bruissements accoutumés dans le vent du soir. C'était immobiliser la Révolution au seuil de la forêt incertaine et obscure $»^{121}$.

Dans l'image que Jaurès donne de l'Allemagne à la fin du xvIII siècle, les «jacobins» occupent une place importante. Notre auteur s'expose ainsi au reproche fait par Reichardt à une certaine historiographie insistant trop, selon lui, sur les «jacobins» lorsqu'elle recherche des éléments révolutionnaires dans l'Allemagne des années 1790 : «llest pourtantprouvédepuisAlbertSoboulauplustardquec'estsurtoutlemouvement des sans-culottes qui a vraiment procuré auxjacobins un soutien social plus large et accéléré en France le processus révolutionnaire ${ }^{122}$. Or, dans les nombreuses pages où Jaurès parle des « jacobins »allemands réunisautourdeForsteràMayenceen 1792-1793, ilnementionnelesmouvements de sans-culottes de cette ville que pour les dénigrer. Le soulèvementdesartisansmayençaisdeseptembre 1790estprésentécomme « des mouvements ineptes », des " désordres misérables » issus de « la grande querelle des ouvriers de métier qui, un soir, dans une auberge, avaient enlevé des filles réservées aux artisans ${ }{ }^{123}$. Reichardt voit par contre dans lesmêmesévénementslapreuvedelaprésenced'une « consciencepolitisée dans les classes moyennes $»^{124}$.

Rudolf Vierhaus a lui aussi souligné que le radicalisme allemand en 1789 n'était pas seulement de type « jacobin », que beaucoup d'Allemands cultivés, dont les grands penseurs, étaient certes très politisés et s'intéressaient aux problèmes sociaux mais que cette forte politisation s'accompagnait rarement d'un désir de révolution, vu que les élites cultivées pensaient que la société d'Ancien Régime pouvait se réformer lentement d'elle-même ${ }^{125}$.Vierhaus confirmefinalementainsilathèsecentrale de Jaurès duradicalismethéoriqueet delatimiditépratiquedespenseurs

(121) Ibid., p. 243 pour toute la métaphore.

(122) Rolf REICHARDT, op. cit., p. 324-325 (traduit par nous).

(123) HSRF, tome IV, p. 171.

(124) Rolf ReICHARDT, op. cit., p. 325 (traduit par nous).

(125) Cf. Rudolf VierHaus, op. cit., particulièrement p. 5-6 
allemands en 1789, «schizophrénie » qui semble un fait culturel marquant sous différentes formes l'Allemagne ${ }^{126}$ jusqu'en 1945, date à partir de laquelle l'idéalisme valorise de plus en plus l'ingérence dans les affaires publiques, exigée même du bon citoyen, si bien qu'aujourd'hui la cloison entre idéal et action semble tombée ${ }^{127}$.

Pour ce qui est du choix de Jaurès, philosophe de formation, de s'occuperprincipalementdecequepensaientlesgrandsespritsdesannées 1790, il constitue certes une particularité de l'approche jaurésienne du radicalisme allemand mais peut aussi être critiqué comme donnant une image partielle de l'Allemagne de l'époque. On pourrait se poser à son sujet la même question que Godechot en 1951 dans sa recension du classique de Jacques Droz, L'Allemagne et la Révolution française ${ }^{128}$ : « Mais dans quelle mesure l'élite intellectuelle représentait-elle les sentiments des masses $»^{129}$ ?

Même si, dans les pages que Jaurès dédie à l'Allemagne dans son Histoire socialiste de la Révolution française, on peut remarquer différents partis pris et oublis, l'analyse qu'il fait des causes complexes de la patience allemande en 1789 est globalement confirmée par la recherche historiqueallemande, par exemple parHans-UlrichWehler. Dans son Histoire de la société allemande, il se demande lui aussi pourquoi il n'y a pas eu de révolution en Allemagne et arrive à des conclusions semblables : émiettement politique, soumission de tous à la noblesse et au clergé régnants, répugnance des bourgeois à l'action politique violente, flegme général d'origine à la fois rationnelle et religieuse ${ }^{130}$. Wehler ne rajoute qu'une seule cause ignorée de Jaurès : les paysans allemands à l'ouest de l'Elbe auraient été à $90 \%$ (en partie) propriétaires de leurs terres, ce qui aurait créé un climat social plus serein qu'en France où les paysans ne l'auraient été qu'à $35 \%{ }^{131}$. C'est-à-dire que le despotisme aristocratique aurait moins pesé sur les paysans allemands que sur les paysans

(126) Walter Grab, «Französische Revolution und deutsche Geschichtswissenschaft » dans Jürgen Voss (Hrsg.), op. cit., p. 300-322.

(127) Cf notre article « Des profondeurs de l'âme à la chair du quotidien. Deux images de l'Allemagne en France : "L'âme allemande" (1933) de Louis Reynaud et "Cousins par alliance" (2002) de Béatrice Durand », Allemagne d'aujourd'hui, nº 182 (octobre-décembre 2007), p. 146.

(128) Jacques Droz, L'Allemagne et la Révolution française, Paris, PUF, 1949.

(129) Jacques GoDECHOT, op. cit., p. 16. La même critique s'appliquerait aux deux autres classiques de la thématique "L'Allemagne et la Révolution » que sont entre Jaurès et Droz: G.P. Gooch, Germany and the French Revolution, London, Frank Cass \& Co,1965 [1920] et Alfred STERN, Der Einfluss der Französischen Revolution auf das deutsche Geistesleben, Stuttgart, Cotta, 1928.

(130) Hans-Ulrich WeHLER, op. cit., p. 353-362.

(131) Cf. Albert Soboul, La Révolution française I De la bastille à la Gironde, Paris, Gallimard, Coll. Idées, 1968, p. 242. 
français. L'Allemagne aurait réussi, grâce à ses " despotes éclairés 》, à modérer l'arbitraire de la noblesse, ce que semble confirmer Jürgen Voss montrant la résistance, parfois violente, des paysans badois aux émigrés français autour du prince de Condé s'imaginant qu'il pouvait se conduire en Allemagne defaçon aussiarbitrairequedanslescampagnesfrançaises avant $1789^{132}$. La « langueur » allemande s'expliquerait-elle finalement surtoutparlefaitquela sociétéallemandede 1789étaitmoinsinjusteque la société française?

Mais si Jaurès souligne les différences entre une mentalité allemandecompliquéeetunementalitéfrançaiseplus directe, ilgardeenvue l'unité fondamentale de l'être. II ne reste jamais englué dans la théorie essentialiste, largement répandue en 1900 des deux côtés du Rhin, des «âmes » nationales enfermant à jamais les peuples dans des différences immémoriales. Pour lui, le véritable idéalisme est celui qui sait à la fois être " profond », à l'allemande, et " actif », à la française, quand les circonstances historiques l'exigent, c'est « la fusion de l'esprit allemand et de l'esprit français ${ }^{133}$ que, à la différence de ses contemporains, il juge possible. En témoignent Forster, Fichte, Hegel et Schelling ou encore ce révolutionnaire allemand peu connu (Venedey) qui, selon le récit de son fils, adorait lire L'esprit des lois de Montesquieu et chanter une chanson mêlant l'Hymne à la joie de Schiller et la Marseillaise ${ }^{134}$. Et Jaurès, un peu mélancolique, de rêver en 1900 à une nouvelle union entre les deux pays : "Quand donc les deux peuples retrouveront-ils, au souvenir deces heures sacrées, la force de refaire leur union $»^{135}$ ?

Éric GUILLeT

Département d'Etudes Germaniques Université de Nantes

1 Quai de Tourville, 44000 Nantes

eric.guillet@univ-nantes.fr

(132) Jürgen Voss, « Baden und die Französische Revolution » dans Jürgen Voss, op. cit., p. 107.

(133) HSRF, p. 141.

(134) Ibid., p. 194-195.

(135) Ibid., p. 141. 